\title{
EL PAPEL DE LAS DISCIPLINAS CIENTÍFICAS EN LA EDUCACIÓN
}

\author{
Evandro AGAZZI \\ Dipartimento di Filosofia. Universita di Genova
}

\section{La ciencia en la cultura actual}

Nuestro tiempo presenta muchas paradojas, y entre estas una muy interesante que concierne al lugar que ocupa la ciencia dentro de nuestra cultura: este lugar es muy reducido, sobre todo si lo comparamos con el gran peso que la ciencia tiene dentro de la vida de nuestra civilización. Para precisar el sentido de nuestra afirmación tenemos que decir que no entendemos el término "cultura" en su sentido sociológico, según el cual la cultura es el conjunto de formas de vida que caracterizan a un cierto grupo social (y, por lo tanto, entran en dicha cultura las maneras de vestir, de cocinar los alimentos, de celebrar las bodas, de trabajar la tierra, así como las costumbres, las convenciones sociales, las instituciones, las prácticas religiosas, etc.). Queremos, al contrario, considerar la cultura según su sentido común y ordinario, que se utiliza, por ejemplo, cuando decimos que una persona es "culta». En este sentido la cultura (de un individuo y también de un grupo) consiste en un sistema rico y articulado de conocimientos interiorizados, que han madurado hasta al nivel de una síntesis original, la cual se expresa en un cierto número de ideas características y visiones intelectuales, que proporcionan criterios de juicio para interpretar y evaluar cosas, eventos, situaciones, y para orientar nuestras decisiones y acciones.

Adoptando esta noción de cultura, no es difícil darnos cuenta, por un lado, que la ciencia (especialmente gracias a sus aplicaciones tecnológicas) influye poderosamente en todas nuestras formas de vida, ya que nos encontramos completamente inmersos en un contexto de realidades artificiales que deter- 
minan hasta los más pequeños detalles de nuestra existencia cotidiana (de manera que lo artificial, el producto de la tecno-ciencia, es el verdadero ecosistema del hombre actual). Sin embargo, por otro lado, es igualmente fácil darse cuenta que las síntesis intelectuales, las perspectivas generales que inspiran los juicios de nuestra época no tienen casi ninguna relación con los conocimientos científicos, sino que dependen de concepciones filosóficas, ideológicas, religiosas, están influenciadas por las obras de escritores, novelistas, periodistas, por películas y telenovelas, por propagandas y mensajes de publicidad, mucho más que por la información cientifica de cualquier tipo.

Pocos ejemplos son suficientes para esclarecer nuestra afirmación. Podemos estar de acuerdo que entre las inspiraciones más significativas de nuestra cultura actual se halla un sentido generalizado de la historicidad, una consciencia de la dimensión social de muchos problemas, una fuerte reivindicación de los derechos de libertad, un sentido agudo de la igualdad de los hombres, la búsqueda de nuevos valores de vida y así sucesivamente. Ahora bien, cada uno de estos elementos de nuestra consciencia cultural puede ser reconducido a influencias de una u otra filosoffa, de algunas ideologías, de la evolución del sentimiento religioso, de la enseńanza de ciertos intelectuales, de la difusión de un cierto tipo de literatura, en resumen: de varios sectores de lo que habitualmente se denota como el mundo de las humanidades. Mucho más diff́cil (aunque no totalmente imposible) serfa indicar elementos de igual importancia, dentro de nuestra cultura, que se puedan considerar como dependientes de mensajes procedentes del inmenso campo de las ciencias. Por último consideremos tres hechos relativamente sencillos, pero también muy sintomáticos. Primero: si pronunciamos la expresión "persona de cultura", es muy probable que los primeros ejemplos que nos vengan a la mente sean nombres de filosofos, literatos, historiadores, juristas, más que de científicos, ni siquiera de los de Newton o Einstein, cuya importancia nadie pone en duda. Segundo: cuando algunos científicos (incluso los de reconocido prestigio) quisieron transmitir un mensaje cultural de su ciencia al público, improvisaron como filósofos o ensayistas (y casi siempre con resultados muy modestos, ya que les faltaba la competencia necesaria para formular este mensaje de manera adecuada). Tercero: todos estamos dispuestos a aceptar que una persona culta no puede ignorar quienes eran Homero, Dante, Goethe, Shakespeare, además de sus obras más famosas, tiene que saber de memoria algunos de sus poemas, versos y citas famosas de la literatura universal, tiene que conocer determinados hechos históricos, identificar algunas composiciones musicales y obras maes- 
tras de las bellas artes, etc., pero no nos escandaliza que esta persona culta no conozca quién era Lagrange, no sepa reconocer una fórmula química, ni se acuerde de la fomulacion de teoremas simples y más conocidos como el de Euclides o Tales, no esté a la altura de calcular las raíces de una ecuación de segundo grado, y todo esto a pesar de que haya pasado años estudiando matemática, física o química en el transcurso de su carrera escolar.

\section{Las razones de la inferioridad cultural de la ciencia}

La paradoja que hemos discutido arriba tiene unas razones que nos permiten explicarla. Una primera y fundamental razón consiste en una postura de desinterés recíproco que los representantes de las disciplinas científicas y humanísticas normalmente manifiestan, no en el sentido superficial que ellos no tengan ninguna curiosidad para lo que se hace en el otro campo (a veces dicha curiosidad existe), sino en el sentido de que ellos no perciben ninguna necesidad de tomar en cuenta los conocimientos y métodos de trabajo que se hallan en el otro campo para trabajar mejor en el propio. Simplemente se ignoran. Esta postura es la consecuencia de una separación entre los dos campos, de la cual queremos ocuparnos más adelante. Pero ya podemos señalar que esta postura se acompaña a menudo de una mezcla de sentimientos de superioridad y inferioridad. Muchas veces los científicos se sienten orgullosos de su ciencia, que es objetiva, rigurosa, produce un incremento de saber, proporciona conocimientos fiables y útiles, y consideran a los humanistas como personas que se contentan con argumentos imprecisos, proceden más retóricamente que críticamente, se pierden en disputas de palabras, nunca llegan a resultados compartidos y estables y no ofrecen nada concretamente útil. Pero, por otro lado, el científico, cuando encuentra problemas de orden general o que implican evaluaciones no estrictamente metodológicas, o incluso que conciernen a la justificación y la fundamentación de sus mismos métodos disciplinarios, puede percibir un cierto malestar intelectual y constatar que sus competencias estrictamente especializadas no le proporcionan una ayuda adecuada. A veces puede estar tentado de sacudiese estos problemas, diciendo que están mal puestos, que no le interesan, que entran en la esfera incontrolable de lo emocional e irracional, pero se da cuenta que esta actitud no basta para eliminarlos, y que la sociedad (y su misma consciencia personal) sigue planteándolos y que otros terminan por hacerse cargo de ellos y proponer soluciones que, tarde 
o temprano, se demuestran eficaces e incluso condicionan el ejercicio de la misma investigación científica. En el caso de los humanistas, su sentimiento de inferioridad es determinado en parte por el hecho de que ellos no deberían conocer las ventajas de la práctica científica que ya hemos mencionado, pero aún más por el inmenso prestigio social del cual goza hoy la ciencia, la cual parece absorber totalmente el plano de lo que propiamente se entiende como "saber" y, además, traduce este saber en un "quehacer" eficaz en todos los sectores de la vida. Se admite también comúnmente que para emprender una carrera científica se necesitan más capacidades intelectuales y un mayor esfuerzo que las que se necesitan para el estudio en las carreras humanísticas, de manera que las ciencias absorben también las mejores energías intelectuales de nuestra época y sus adeptos parecen constituir una verdadera elite intelectual. Por otro lado, un sentimiento de superioridad caracteriza a menudo los humanistas en cuanto, aceptando la imagen de la ciencia proporcionada por muchas filosofías de nuestro siglo, la consideran como un conjunto de visiones parciales que nunca alcanzan la verdad auténtica de las realidades que estudian, y que tienen únicamente una utilidad práctica. Además, muchos piensan que las humanidades poseen una aristocracia intrínseca porque se dirigen al mundo del hombre y de la civilización, a la esfera de los valores espirituales, morales, artísticos, al ejercicio desinteresado de la razón y de las capacidades creativas del hombre, lo cual se considera la única forma de alcanzar una formación armónica del ser humano.

\section{El camino para recuperar la función cultural de la ciencia}

Hay una raíz común a los diversos fenómenos culturales que hemos mencionado, y consiste en una imagen falseada de la ciencia, la cual explica al mismo tiempo su gran prestigio social y su esterilidad cultural. Según esta imagen la ciencia es un poderoso conjunto de conocimientos eficaces y a los ojos de la mayoría, se identifica con la tecnología (en efecto, cuando se habla de progreso científico, de conquistas de la ciencia, casi todos pensamos, y con mucha admiración, en una gran cantidad de ejemplos que en realidad son realizaciones tecnológicas). Lo que se pierde de vista es el valor de la ciencia en cuanto saber, en cuanto puro y simple conocimiento objetivo y riguroso. Por lo tanto, si se considera la ciencia como un catálogo ordenado de instrucciones prácticas para conseguir resultados concretos, su 
impacto cultural se reduce a casi nada, ya que nadie piensa buscar ideas e inspiraciones dentro de un libro de recetas. A menudo se inculpan por haber difundido esta imagen triunfalista y al mismo tiempo empobrecida de la ciencia a varias filosofías de nuestro siglo (idealistas, espiritualistas, existencialistas) que se afirmaron como reacción al positivismo del siglo pasado. Pero no debemos de olvidar que los positivistas, como E. Mach, fueron los primeros que negaron a la ciencia la tarea de proporcionar conocimientos, atribuyéndole únicamente el valor de elaborar esquemas convencionales útiles desde un punto de vista práctico. Hoy en día, la mayoría de las filosofías de la ciencia de tipo anti-realista e instrumentalista, siguen colocándose dentro del enfoque neopositivista. Su diferencia con respecto a las filosofias anti-científicas es que no atribuyen a otras formas de pensamiento (por ejemplo a la filosofía) el privilegio de alcanzar conocimientos. De hecho, ni siquiera la ciencia goza de este privilegio ya que está muy centrada en su utilidad práctica. Si así están las cosas, el primer paso que hay que dar es una completa recuperación de la intención y del valor cognoscitivo de la ciencia, hay que volver a considerarla como expresión de la incansable labor del hombre para conseguir la verdad, utilizando sus mejores capacidades de investigación empírica y de razonamiento crítico. Desde este punto de vista se puede ver que la ciencia ha sido capaz, y lo es todavía, de conseguir muchas verdades, aunque éstas estén limitadas a los particulares ámbitos de objetos que cada disciplina científica puede investigar, utilizando determinados instrumentos y métodos. La que fue considerada como una crisis de la verdad científica a finales del siglo pasado era en realidad la crisis de una verdad que se pretendía absoluta y total (y que por lo tanto quedaba derrotada si se descubrían nuevos hechos fuera del campo limitado dentro del cual ella había sido alcanzada y con los cuales no resultaba compatible). Para llegar a una toma de consciencia de este tipo es necesario no dejarse deslumbrar por las maravillas de las conquistas de la ciencia y la tecnología, sino darse cuenta de la complejidad del trabajo de investigación cientifica, del alcance y de los límites inherentes a sus métodos e instrumentos, de la componente histórica que siempre caracteriza a estos métodos e instrumentos y que se refleja también en los resultados conseguidos. Está claro que todo esto no se produce espontáneamente, sino que tiene que ser el resultado de una educación científica correctamente practicada. Aquí nos encontramos frente a una situación nuevamente paradójica: todos estamos convencidos, por una especie de opinión común pasivamente aceptada, 
de que la ciencia es la forma más perfecta del pensamiento crítico y riguroso y, por lo tanto, creemos que una educación científica debe, por sí misma, producir en los que la reciben una actitud intelectual de rigor y crítica racional. En verdad estamos muy lejos de esto. Las disciplinas en las cuales los estudiantes encuentran diferentes opiniones, discusiones, presentaciones de puntos de vista opuestos que se combaten también mediante argumentos racionales y que, además, abundan en perspectivas históricas son las humanidades. Al contrario, la educación científica consiste casi totalmente en la transmisión de nociones que deben aprenderse en forma básicamente dogmática. Si alguien tiene alguna duda acerca de una noción, un teorema, un resultado de matemática, de física, de química, sabe que no puede poner en duda todo esto, sino que no ha entendido bien las cosas, que su esfuerzo debe consistir en comprender y convencerse de que lo que está escrito en el manual o lo que su maestro le ha presentado es la verdad. En cuanto a la dimensión histórica, queda prácticamente ausente de nuestra educación científica, o se considera casi como una curiosidad o un adorno, ya que se piensa que todo lo verdadero alcanzado por la ciencia pasada se conserva dentro de la ciencia actual y no tenemos necesidad de tomarlo en cuenta en las formas imperfectas de la ciencia de otras épocas. Solamente los que siguen una carrera científica completa y se dedican profesionalmente a la investigación científica llegan a tener en cuenta la naturaleza crítica de la ciencia (a condición de ocuparse de problemas abiertos en la frontera de la investigación), aunque no está garantizado que todos ellos salgan de la visión dogmática que recibieron durante su educación. En conclusión, sólo una robusta integración de una profunda reflexión epistemológica en el cuerpo de la educación científica, y una consideración no puramente anecdótica de la historia de la ciencia pueden ayudar a recuperar el valor cultural de la ciencia. Desde este punto de vista, la historia proporciona una posibilidad muy eficaz para evaluar la presencia cultural de la ciencia, puesto que ella muestra con la evidencia de los hechos (bien entendidos y explicados) dos cosas igualmente importantes: que la ciencia es un factor de la historia muy poderoso, y que es también un producto de la historia, al igual que las instituciones políticas, las ideologías, las doctrinas filosóficas, las obras de la literatura y del arte. Este propósito es más fácil de enunciar que de llevar a cabo, porque desde hace mucho tiempo nos hallamos dentro de una verdadera separación de las ciencias y las humanidades. Vamos ahora a considerar las razones de esta separación. 


\section{La separación entre ciencias y humanidades}

La noción de ciencia siempre ha sido utilizada en la tradición occidental, desde los tiempos de los griegos, para indicar el saber en su forma más adecuada y en cualquier campo que este saber pudiese realizarse. Con el Renacimiento surgió la ciencia moderna, caracterizándose en un primer momento como una manera menos ambiciosa, pero al mismo tiempo más segura, para investigar la naturaleza fisica. Los grandes éxitos conseguidos por esta ciencia natural tuvieron como consecuencia promover las nuevas formas y métodos de investigación, que ella había aplicado brillantemente, al rango de nuevo paradigma de la racionalidad. Una racionalidad que, además de su seguridad (garantizada también por el uso de los instrumentos matemáticos), ofrecía la gran ventaja de permitir la constitución de un saber objetivo, y esto correspondía a una exigencia muy acuciante. De hecho, la filosofía moderna había descubierto el mundo de la subjetividad, pero se veía obligada a superar esta misma subjetividad cuando se trataba de garantizar un conocimiento válido. Ahora bien, la nueva ciencia natural proporcionaba ejemplos estupendos de conocimiento intersubjetivo. Se entiende así como la Razón, tan celebrada por los representantes de la filosofía de las Luces, era una razón universal en el sentido de racionalidad científica. La consagración de esta concepción se encuentra en la filosofía de Kant, el cual restringió la esfera del conocimiento a lo que se puede alcanzar utilizando métodos que se inspiran directamente en los de las ciencias físico-matemáticas, y al mismo tiempo trató de protege las certezas morales y metafísicas, y los juicios de valor, conectándolos a la esfera de la moralidad, que se funda en la libertad absoluta del sujeto. Este tipo de fundamentación quedaba, según su pensamiento, dentro de la esfera de la razón, pero no dentro de la esfera del conocimiento. El camino triunfal de las ciencias naturales a lo largo del siglo XIX tuvo como consecuencia que la racionalidad fuera reducida únicamente a la racionalidad científica, y que todo el mundo de la subjetividad fuera dejado a otras formas de pensamiento, que se consideraban irracionales en cuanto ligadas al sentimiento y las emociones. Se consolidó, de esta manera, una efectiva separación entre ciencia y valores, que caracterizó la mentalidad positivista y que, en un primer momento, significó una separación entre ciencias y humanidades. Sin embargo, en un segundo momento, se consideró que el mismo mundo del hombre podía ser investigado científicamente y surgieron las diferentes ciencias humanas (historia, sociología, psicología, antropología, economía y lingüística). Aunque sus 
especialistas llegaron pronto a defender su cientificidad como algo diferente de la de las ciencias de la naturaleza, y hasta aceptaron que en estas ciencias no se podía evitar una referencia a los valores (Max Weber), sin embargo, insistieron en que las ciencias sociales debían abstenerse absolutamente de juicios de valor (es decir, debían ser "value-free», como las ciencias naturales). Este imperativo metodológico se justificaba con la tesis de que los juicios de valor dependen de una opción subjetiva que no se puede fundamentar racionalmente. Cuando se produjo al final del siglo XIX la crisis de las ciencias exactas, las filosofías anti- positivistas, además de descalificar a la ciencia, atribuyeron a las humanidades (concebidas como disciplinas no cientificas), rango preferente, y de esta manera confirmaron la separación entre ciencias y humanidades, limitándose a invertir su orden jerárquico. En nuestra época nos hallamos todavía bajo el influjo de esta separación, y los diferentes proyectos de educación que se elaboran en el mundo muy a menudo tratan de promover las disciplinas cientfficas a expensa de las humanidades, o al revés. Se trata de una situación que es urgente superar, ya que ambas disciplinas son igualmente esenciales para la formación del hombre, como se puede fácilmente argüir, a partir de las consideraciones hechas en párrafos anteriores referidas a las posturas paradójicas de los científicos y los humanistas.

\section{El valor humanístico de la ciencia}

Un camino eficaz para salir de la situación mencionada es, por un lado, darse cuenta de los valores humanísticos que se hallan dentro de una concepción y una práctica adecuadas de las ciencias y, por otro lado, tener en cuenta las dimensiones científicas que se hallan dentro de las humanidades. Vamos a empezar con el primer aspecto. Para entender el valor humanístico de la ciencia hay que superar, como ya hemos visto, la visión esencialmente utilitarista y pragmática que, a menudo, se tiene de ella y concentrarse en su estructura cognoscitiva. Desde este punto de vista, la ciencia se presenta como una de las formas más avanzadas del pensamiento humano (es decir, como una de las características que constituyen lo especifico del hombre). Esta fuerza del pensamiento no se revela tanto en el descubrimiento de nuevos hechos, cuanto en el esfuerzo de explicarlos, es decir de dar la razón de por qué son asi, independientemente de que ellos puedan ya ser ciertos. Este es el auténtico sentido de 
la formulación de hipótesis y teorías, las cuales (contrariamente a lo que han afirmado los neoempiristas y el mismo Popper) no se confunden con las leyes científicas. Una ley empírica es el resultado de una generalización inductiva y, aunque pueda ser confirmada por el descubrimiento de nuevos hechos dentro del mismo campo de investigación, no por esto debe calificarse como hipótesis. La verdadera hipótesis es algo que se admite para explicar las leyes empíricas, y es diferente del conjunto de los hechos observados no por ser general (es decir, cuantitativamente más extensa), sino por postular características de la realidad que no se observan dentro de los hechos (es cualitativamente diferente). Por esto se requiere una verdadera capacidad de invención creativa, que proporcione nuevas ideas y modelos, de manera no muy diferente a la creatividad que se encuentra en la filosofía y las artes. La ciencia, como hemos visto, se caracteriza por ser un saber objetivo y riguroso. Esto implica que hasta las más brillantes hipótesis y teorías tienen que someterse a un severo análisis crítico, lo que significa básicamente dos cosas. En primer lugar, se debe averiguar la compatibilidad lógica entre las nuevas hipótesis y las otras proposiciones (factuales o teóricas) ya admitidas en el tejido de las ciencias. En segundo lugar, no se debe perder de vista que las hipótesis se legitiman por el hecho de que permiten explicar (es decir deducir correctamente) los hechos observados, y la lógica elemental nos enseña que el hecho de permitir la deducción de consecuencias verdaderas nunca es garantía suficiente para afirmar la verdad de las premisas. Por lo tanto, el constructo hipotético y teórico siempre está abierto a una posibilidad de refutación y exige una constante vigilancia crítica. Acostrumbrarnos a pensar y practicar las ciencias según este espíritu es una preciosa escuela de pensamiento crítico cuyo valor humanístico es evidente. No solamente porque es clara la utilidad de adoptarlo también en el campo de las humanidades (como de hecho ocurrió cuando éstas intentaron tomar un talante cientifico), sino porque es también muy clara su importancia como actitud general en la manera de pensar de los hombres. La fecundidad de esta manera de pensar no se acaba en el hecho evidente de contrastar a la perniciosa actitud dogmática tan difundida, sino que resulta más clara si se valora adecuadamente la consciencia de lo que significa el trabajar bajo hipótesis. En esto se hallan tres elementos importantes. En primer lugar, se trata de reconocer la necesidad de las hipótesis en cualquier investigación. El que estudia un objeto empieza por la hipótesis sobre el lugar donde dicho objeto se encuentra, luego sobre sus características, hasta que la hipótesis resulta confirmada. Pero la misma cosa ocurre cuando se busca la correcta traducción de un texto, la 
correcta interpretación del pensamiento de un autor, la solución de un problema matemático, la reconstrucción de un hecho histórico. Sin embargo (y este es el segundo elemento) la hipótesis tiene que ser tomada como un modesto farol de proa que nos permite explorar un corto espacio de nuestro camino, y nunca como el faro del puerto hacia el cual dirigirnos con plena seguridad (lo que, fuera de esta metáfora, equivaldría a forzar los datos para que apoyen a nuestra hipótesis tomada como una verdad apriorísticamente aceptada). Al final ( $y$ este es el tercer elemento), hay también que evitar el riesgo de dejar caer una hipótesis fecunda por escasez de agudeza, por no haber sabido descubrir los nexos que le permiten explicar los hechos que nos interesan. Está bastante claro que este equilibrio delicado entre tenacidad y provisionalidad, invención, rigor y prudencia es un hábito intelectual que se revela precioso en las más diferentes circunstancias de la vida: en tal sentido él posee un valor humanístico y cultural de primera importancia.

Mencionamos ahora una última consecuencia de esta valoración humanista del espiritu cientifico. Si se lo toma en serio y con plena coherencia, nos lleva a superar aquella patología de la ciencia que se llama habitualmente "cientificismo". En efecto, si se entiende bien en qué consiste la vertiente crítica de la ciencia, se ve que ésta consiste en considerar la ciencia como un saber abierto. A menudo se acepta esta caracterización en el sentido de que cada teoría científica tiene que quedarse abierta a la posibilidad de refutaciones. Esto es verdad, mas esta característica descansa, en último análisis, en el hecho de que cada saber cientifico es limitado (depende de los límites inherentes a los instrumentos de investigación utilizados, del cuadro teórico adoptado, de los criterios admitidos para controlar las hipótesis. etc.). Estos son al mismo tiempo límites históricos de la ciencia en cada época, pero también límites de la racionalidad científica en cuanto tal, y la ciencia estará realmente abierta cuando reconozca estos límites y se abra a la consideración de realidades y problemas que no cabe dentro de sus perspectivas y procedimientos limitados. El sentido de la vida, el saber lo que es bueno o malo hacer, las motivaciones de la acción humana, el valor de la persona, la determinación de derechos y deberes, la existencia o no existencia de realidades diferentes de las que se pueden constatar empíricamente son todas ellas cuestiones y problemas profundamente humanos, y que no pueden ser ignorados por una ciencia humanísticamente abierta. Esto no significa que ella tenga que hacerse cargo de estos problemas ni que pretenda solucionarlos mediante el uso exclusivo de sus instrumentos y métodos (ésta es justamente la postura del cientificismo), sino que 
debe tomarlos en cuenta y reconocer que hay otras formas de pensamiento humano que se preocupan especificamente de ellos. Muchas de estas formas coinciden con varias disciplinas humanísticas, y por lo tanto resulta claro que una ciencia humanísticamente inspirada reclama, en un cierto sentido, la colaboración con las humanidades, lejos de sentirse separada de ellas.

\section{Las dimensiones científicas de las humanidades}

No estamos interesados, en este momento, en considerar cómo los contenidos del conocimiento científico han estado, durante siglos, a la base de las expresiones de las humanidades en la cultura occidental (como en la poesía de Dante o Milton, o en el pensamiento de una verdadera legión de filósofos antiguos y modernos), ni tampoco ver cómo muchos grandes científicos estuvieron sinceramente interesados por las letras, las artes, la filosofia, la teología, y supieron sacar, de este interés por las humanidades, importantes estímulos para sus concepciones científicas. Ya hemos visto que la separación entre ciencias y humanidades es un fenómeno histórico relativamente reciente, ligado a la alternancia y oposición entre un enfoque positivista y otro anti-positivista. Nuestra intención se limita a indicar cómo las componentes fundamentales del espíritu cientifico y de su racionalidad se han introducido en el campo de las humanidades de forma fructifera. Para entender correctamente esta afirmación hay que darse cuenta de que el concepto de ciencia no tiene un sentido unívoco, sino analógico, es decir, cada disciplina cientifica es tal en la medida en que realiza un conocimiento objetivo y riguroso, pero los requisitos de dicha objetividad y rigor no son necesariamente los de las ciencias exactas. Cada ciencia tiene la obligación de proporcionar criterios precisos para determinar intersubjetivamente cuáles son los datos que quiere investigar y explicar, y desarrollar tipos de argumentación rigurosa para proporcionar dichas explicaciones. Sin embargo, está claro que hay diversos criterios mediante los cuales se establecen los datos históricos (búsqueda e interpretación de documentos, restos arqueológicos, monedas, inscripciones, fuentes de varios tipos), filológicos, sociológicos, etnográficos, económicos, jurídicos, etc. En cada caso se necesita también la ayuda de ciertas disciplinas auxiliares y un serio entrenamiento, del mismo modo que un físico tiene que saber manejar instrumentos matemáticos y materiales, y estar entrenado en el uso correcto de los mismos para encontrar e interpretar los datos de su ciencia. 
En cuanto al rigor argumentativo, no es necesario que se exprese en cálculos o deducciones matemáticas: todo el mundo conoce cuán articuladas y rigurosas pueden ser las argumentaciones. jurídicas, o cómo un buen historiador tiene que saber discutir, conectar, compatibilizar diferentes fuentes y documentos (y hasta explicar ciertas discrepancias entre ellos) para justificar sus interpretaciones y explicaciones de los acontecimientos pasados. Lo que hemos dicho arriba acerca del auténtico espiritu científico nos dispensa de insistir en esta ejemplificación y podemos contentarnos con decir que, en la medida en que en las disciplinas humanísticas se hallan problemas de conocimiento, es correcto tratar de alcanzarlo urilizando los criterios de la cientificidad. En general, podemos decir que el orden conceptual, la disciplina intelectual, la precisión del discurso, la claridad de la exposición, el rigor de las argumentaciones, la preocupación de justificar nuestras afirmaciones, la honestidad en reconocer nuestros errores, no son cualidades menos apreciables en cualquiera de las disciplinas humanísticas que en las ciencias exactas (donde su falta es inmediatamente detectada y criticada). Podemos, incluso, decir que el respeto por estas cualidades debería ser un remedio muy saludable contra ciertos defectos que todavía se hallan en las humanidades (tendencia a la retórica, gusto por el oropel, verbosidad, obscuridad de lenguaje que quiere pasar por profundidad de pensamiento, intolerancia de la crítica, etc.). Sin embargo, está claro que las humanidades no pueden limitarse a este aspecto, porque ellas se dirigen muy directamente al mundo de la subjetividad y tienen que ayudar al hombre a desarrollar los diferentes aspectos de ésta según sus personales exigencias de vida, y por lo tanto no pueden limitarse a presentar conocimientos impersonales. De todas maneras hay que distinguir entre la expresión, la expansión, el crecimiento de nuestra subjetividad y el conocimiento de ella. Los primeros aspectos se viven por experiencia directa y se cultivan, precisamente, viviéndolos. Así no se puede enseñar mediante preceptos objetivos a pensar con claridad, a empeñarse moralmente, a creer en Dios o en la libertad, a confiar en la democracia. Lo que una educación humanística puede y debe hacer, en esta dirección es proponer ejemplos, ayudar a hacer experiencias, estimular la reflexión, ayudando a afinar y madurar la cualidades interiores que puedan permitir al sujeto configurar libremente y con plena consciencia su constelación de valores. Todo esto, por otro lado, no excluye que este mundo tan complejo de la subjetividad pueda ser investigado objetivamente, tratando de delinear sus características, sus expresiones históricas, artísticas, institucionales, culturales, analizando los sistemas de ideas y valores en los cuales se ha 
traducido, reflexionando sobre las mismas dinámicas y estructuras de la subjetividad individual. En esta empresa de conocimiento es no sólo posible, sino muy deseable que se aprovechen aquellos estilos de objetividad y rigor que equivalen a aplicar (en el sentido analógico ya presentado) las categorías de la cientificidad. De esta manera se realizaría una apertura no extrínseca de las humanidades hacia las ciencias, simétrica de la apertura de las ciencias hacia las humanidades de que hemos discutido antes, y se promoveria aquella unidad de la cultura que reconoce las distinciones sin transformarlas en separaciones u oposiciones. Además hay que subrayar que la pretensión de cultivar los valores sin preocuparse de conocer las características concretas de las diferentes realidades en las cuales éstos han determinado nuestras formas de vida y de acción equivaldría a volverlos inoperantes o hasta aplicarlos de manera inadecuada o errónea. Lo mismo vale para todo lo que concierne al hombre: es ingenuo pretender concentrarse sobre su mera interioridad, ya que nuestra subjetividad resulta al mismo tiempo de factores interiores y exteriores vanamente interactivos. Por lo tanto, si una tarea importante de las disciplinas humanísticas es la de ayudarnos a conocer al hombre, ellas no pueden pasar por alto los métodos, ni tampoco las aportaciones cognoscitivas de las demás ciencias, para estar a la altura de esta tarea. 